\title{
CIRRUS CLOUDS PROPERTIES DERIVED FROM POLARIZED MICRO PULSE LIDAR (P-MPL) OBSERVATIONS AT THE ATMOSPHERIC OBSERVATORY 'EL ARENOSILLO' (SW IBERIAN PENINSULA): A CASE STUDY FOR RADIATIVE IMPLICATIONS
}

\author{
Ana del Águila ${ }^{1 *}$, Laura Gómez ${ }^{1}$, José Manuel Vilaplana ${ }^{1}$, Mar Sorribas ${ }^{1}$, and Carmen Córdoba- \\ Jabonero ${ }^{1}$
}

${ }^{1}$ Instituto Nacional de Técnica Aeroespacial (INTA), Atmospheric Research and Instrumentation Branch, Atmospheric Observatory 'El Arenosillo’'(Huelva), Spain,*aguilapa@inta.es

\begin{abstract}
Cirrus (Ci) clouds are involved in Climate Change concerns since they affect the radiative balance of the atmosphere. Recently, a polarized Micro Pulse Lidar (P-MPL), standard system within NASA/MPLNET has been deployed at the INTA/Atmospheric Observatory 'El Arenosillo' (ARN), located in the SW Iberian Peninsula. Hence, the INTA/P-MPL system is used for $\mathrm{Ci}$ detection over that station for the first time. Radiative effects of a Ci case observed over ARN are examined, as reference for future long-term $\mathrm{Ci}$ observations. Optical and macrophysical properties are retrieved, and used for radiative transfer simulations. Data are compared to the measured surface radiation levels and all-sky images simultaneously performed at the ARN station.
\end{abstract}

\section{INTRODUCTION}

Cirrus (Ci) clouds play an important role in Earth radiative forcing and Climate Change [1]. The degree of understanding of their climate implication is still low; hence, Cirrus features are widely required in data assimilation by global climatic models. In particular, the contribution of Ci-contrails formed from air traffic is also poorly known. In addition, $\mathrm{Ci}$ cloud properties, both macrophysical and optical, affect the solar radiation levels reaching the ground, enhancing or negating the global warming effect. Thereby, a better understanding of $\mathrm{Ci}$ cloud properties is needed in order to explain their influence in the radiative balance.

Active remote sensing instruments, as lidar systems, are generally used to detect $\mathrm{Ci}$ clouds heights. In particular, a polarized Micro-Pulse Lidar (P-MPL), standard NASA/Micro-Pulse NETwork (MPLNET, mplnet.gsfc.nasa.gov) system, was recently deployed at the INTA/Atmospheric Observatory 'El Arenosillo' (ARN), where flight routes frequently cross over this area as well. This P-MPL has been used for $\mathrm{Ci}$ detection over ARN for the first time; a $\mathrm{Ci}$ cloud case observed over ARN on 28 October 2016 is presented in this work, as reference for future long-term $\mathrm{Ci}$ observations. The aim is to compare the retrieved $\mathrm{Ci}$ optical and macrophysical properties to the surface radiation levels measured at ARN station. Radiative transfer simulations are also performed to study the impact on surface of the Ci clouds. Results can contribute to validation purposes of the next ESA's EarthCARE mission, whose scientific goal is related to the radiation-aerosol-cloud interactions.

\section{METHODOLOGY}

\subsection{Measurement station and lidar system}

The Atmospheric observatory 'El Arenosillo' (ARN) is located at the Southwest Iberian Peninsula $\left(37.1^{\circ} \mathrm{N} 6.7^{\circ} \mathrm{W}, 40 \mathrm{~m}\right.$ a.s.l.), inside a natural protected environment near Doñana National Park and less than $1 \mathrm{~km}$ from the Atlantic coastline. ARN is managed by the Spanish Institute for Aerospace Technology (INTA), and also is a NASA/AERONET site.

The new polarized Micro Pulse Lidar (P-MPL) model 4B (Sigma Space Corp., USA) was deployed at ARN station at the end of September 2016. The P-MPL system is an elastic lidar with a highly-pulsed $(2500 \mathrm{~Hz})$ and low-energy $(\sim 7 \mu \mathrm{J})$ $\mathrm{Nd}$ :YLF laser at $532 \mathrm{~nm}$, operational in full-time continuous mode $(24 \mathrm{~h} / 7 \mathrm{~d})$. Polarization parameters are determined from the co- and crosschannels, obtaining both the parallel and perpendicular range-corrected signals ( $\mathrm{p}-\mathrm{RCS}$ and s-RCS, respectively) by applying [2]. P-MPL 
routine measurements follow the NASA/MPLNET requirements of 1-min integrating time and $75-\mathrm{m}$ vertical resolution. Due to the variability of the Cirrus clouds, registered 1-min lidar profiles are 10-min averaged in order to obtain a relatively good signal-to-noise ratio without neglecting potential $\mathrm{Ci}$ variability.

\subsection{Retrieval of the $\mathrm{Ci}$ properties}

Macrophysical and optical properties of Ci clouds observed on 28 October 2016 (unfortunately, no data are available after noon) have been retrieved by using the procedure described in [3]. In particular, both top and base heights, the thickness, the backscattering ratio (BSR, total-tomolecular backscattering coefficient ratio), the volume linear depolarization ratio $\left(\delta^{\mathrm{V}}\right)$, the $\mathrm{Ci}$ Cloud Optical Depth (CCOD), and the Lidar Ratio (LR) are derived. $\mathrm{Ci}$ clouds are also classified according to their CCOD: sub-visual (svCi, CCOD < 0.03), semi-transparent (stCi, CCOD: 0.03-0.3) and opaque (opCi, CCOD > 0.3).

\subsection{Solar radiation measurements}

Solar radiation data were obtained by a set of radiometers installed on a Kipp\&Zonen 2AP solar tracker. The Global (G) and Diffuse (F) radiations were measured by using two Kipp \& Zonen CMP21 pyranometer (spectral range: $285-2800$ $\mathrm{nm}$ ), shadowing the one measuring the diffuse component. A Kipp \& Zonen SHP1 pyrheliometer $(200-4000 \mathrm{~nm})$ and a YES UV.B-1 pyranometer $(270$ - $400 \mathrm{~nm})$ were used to measure, respectively, the Direct (D) and Erythemal effective UV (UV-Ery) radiations. Data were also 10-min averaged, as for the lidar profiles. Those instruments are regularly calibrated with traceability to the World Radiation Reference.

\subsection{Radiative transfer simulations}

Radiative transfer simulations were performed to calculate the (spectrally-integrated) global $(\mathrm{G})$, direct (D) and diffuse (F) irradiances on the surface within the spectral range from 285 to 2800 $\mathrm{nm}$, as well as the Erythemally-weighted UV irradiance. The radiative transfer model (RTM) used for these calculations was the pseudospherical SDISORT code [4], using the US standard atmosphere as input for the model. In order to determine the impact of the Cirrus clouds on the surface radiation levels, two scenarios were considered: (1) No Ci clouds occurrence; and (2) the presence of an homogenous layer of $\mathrm{Ci}$ clouds (until noon), whose lidar-derived altitude and optical depth were input parameters into the RTM.

\section{RESULTS}

\subsection{Optical and macrophysical properties of Ci clouds}

A layer of $\mathrm{Ci}$ clouds was observed over ARN on 28 October 2016 (unfortunately, no data were available from 11:45 UTC on). The vertical BSR $(\mathrm{R})$, volume linear depolarization ratio $\left(\delta^{\mathrm{V}}\right), \mathrm{Ci}$ Cloud Optical Depth (CCOD), and Lidar Ratio (LR) were derived. Their temporal evolution is shown in Figures $1\left(\mathrm{R}\right.$ and $\delta^{\mathrm{V}}$ ) and 2 (CCOD and $\mathrm{LR}$, together to their standard deviation).

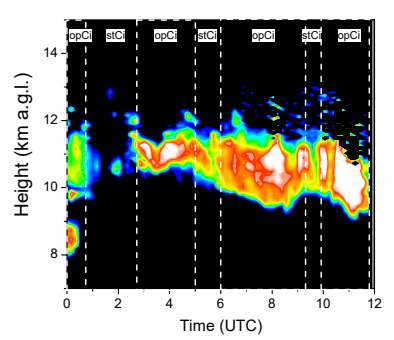

(a)

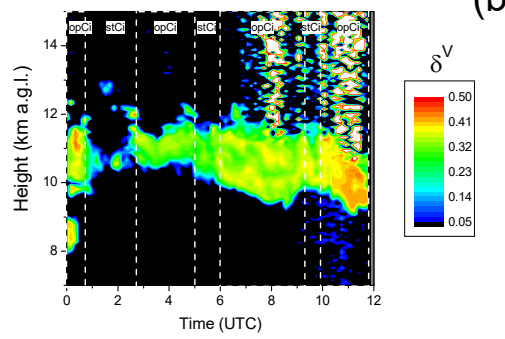

(b)

Figure 1 Temporal evolution of a Ci case observed over ARN on 28 October 2016 in terms of: (a) Backscattering ratio (R), and (b) Linear Volume Depolarization Ratio $\left(\delta^{\prime}\right)$. Ci CCOD-type is also marked at the top.

On the top of all figures, the $\mathrm{Ci}$ predominant CCOD-type category for consecutive Ci layers is labelled. Seven Ci groups are found, alternating between $\mathrm{stCi}$ and opCi categories; but no $\mathrm{svCi}$ were observed. During the overall observational period (almost $12 \mathrm{~h}$, first half of the day), opCi clouds were more frequent $(68 \% / \sim 8 \mathrm{~h})$ than $\mathrm{stCi}$ $(32 \% / \sim 4$ h) (see Fig. 1). In particular, CCOD values higher than 1 are retrieved in the daytime intervals: 07:50-08:20 UTC, and 10:30-11:30 UTC, where the highest R and $\delta^{\mathrm{V}}$ values are found 
(see Figs. 1 and 2-up). Regarding LR, lower values are found for opCi (around 20-40 sr) than for stCi (around 30-50 sr) (see Fig. 2-center). In the overall period, the mean values of CCOD and LR are $0.8 \pm 0.7$ and $28 \pm 6 \mathrm{sr}$, respectively, being $1.2 \pm 0.8$ and $25 \pm 5 \mathrm{sr}$, respectively, for daytime.

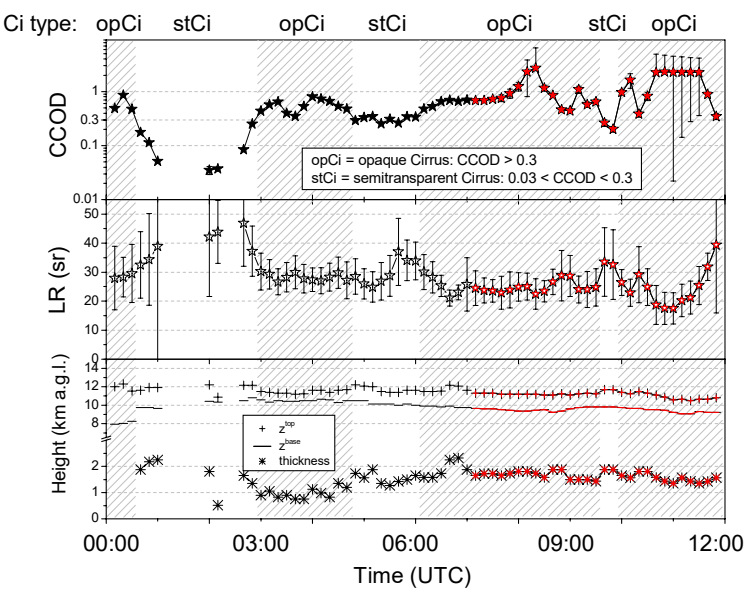

Figure 2. Optical and macrophysical properties: (UP) Cirrus Cloud Optical Depth (CCOD, full stars); (CENTER) Lidar Ratio (LR, open stars); and (DOWN) both top and base heights ('+' and '-' symbols, respectively) and thickness (stars) of the Ci cloud layers. Ci CCOD-type is labelled at the top and marked by white (stCi) and shaded (opCi) bands. Daytime interval is shown in red.

$\mathrm{Ci}$ clouds are found just below the tropopause height-level (around $13 \mathrm{~km}$ height), showing the top and base boundaries of the Ci layers ( $\mathrm{z}^{\text {top }}$ and $\mathrm{z}^{\text {base }}$, respectively) together with their thickness along the day until noon in Figure 2-down. $\mathrm{z}^{\text {base }}$ ranges between 8.0 and $10.8 \mathrm{~km}$ above ground level (a.g.1.), and $\mathrm{z}^{\text {top }}$ ranges between 10.7 and $12.3 \mathrm{~km}$ a.g.l. Their thickness varies between 0.5 and $2.3 \mathrm{~km}$. Temperatures at the $\mathrm{Ci}$ top levels (data not shown) are lower than that threshold determined for ice cloud formation $\left(-38^{\circ} \mathrm{C}\right)$.

\subsection{Surface radiation levels}

Surface solar radiation measurements and all sky images (not shown) show a good agreement with the lidar retrievals. Global $(\mathrm{G})$, diffuse $(\mathrm{F})$, and direct (D) components of the total integrated solar spectrum (including the Erythematically-weighted radiation, UV-Ery) reaching the ground are examined. The discussion will be addressed for the first half of the daytime since lidar measurements are only available until noon (see Figure 3, shaded area).

The solar radiation intensity increases (decreases) with the solar elevation (zenithal angle) reaching the maximum at noon. Short wavelengths are rather less affected by $\mathrm{Ci}$ clouds than those in the visible range because the diffuse/global ratio is larger in the shortwave range and consequently less sensitive to the presence of these high $\mathrm{Ci}$ clouds in the sun path [5]. Indeed, the effect in the Erythemal radiation (UV-Ery in Fig. 3) is only significant when the CCOD is large enough and $\mathrm{Ci}$ clouds are in the sun path. This is in agreement with lidar results, since a dense opCi occurrence with CCOD values around 2 is observed in the interval 10:30-11:30 UTC, affecting clearly all the radiation components, and especially evident for the UV-Ery radiation (see Figs. 2-up and 3).

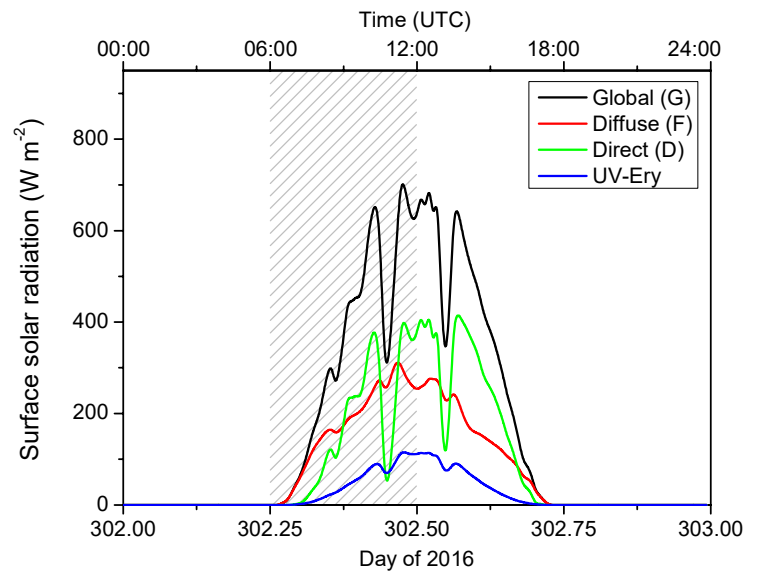

Figure 3. Daily evolution of the measured Global $(G)$, Diffuse (F), Direct (D) and Erythemal effective UV (UV-Ery) radiations on the surface on 28 October 2016. Shaded area corresponds to the simultaneous lidar measurement period.

However, the most sensitive to these Ci clouds is the direct component, where larger variations are also observed, even with CCOD $<2$ (see Figs. 2up and 3). This different behaviour between the Erythemal radiation (UV-Ery) and the direct total solar component (D) suggests the presence of Cirrus instead of lower clouds attenuating both diffuse and direct components of the radiation. The diffuse/global ratio, as expected, increases under Ci cloudy skies [5]. 
The fraction of overcast sky and the density of the $\mathrm{Ci}$ clouds changes from morning to noon and, consequently, the evolution of the solar radiation (as the solar zenith angle decreases) departs from the typical 'bell' that would be expected in case of clear sky conditions (see Fig. 4). In addition, it is observed that this solar affected by $\mathrm{Ci}$ clouds 'bell' is different between measurements and simulations. However, the daytime strongest attenuation measured at around 11:00 UTC coincides with a quite dense $\mathrm{Ci}$ cloud (CCOD 2), as before exposed, detected by the lidar in front of the sun, and this sun occultation strongly affected both UV and total radiation budget.
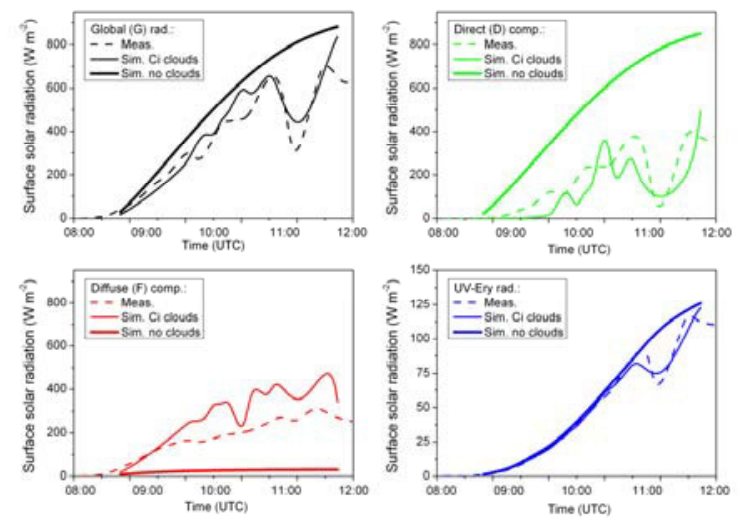

Figure 4. (From left to right, and up to down): Surface Global ( $G$, black), Direct (D, green), Diffuse ( $F$, red), and Erythemal effective UV (UV-Ery, blue; note the different solar intensity-axis scale) radiations during the first half part of the daytime on 28 October 2016, as obtained from: measurements (shaded line), and simulations under both no clouds (thicker solid lines) and $\mathrm{Ci}$ clouds presence (thinner solid lines).

Therefore, differences between measurements (registered under a given cloud cover state of the sky) and simulations (performed using the lidar optical products derived for $\mathrm{Ci}$ detected just above the lidar sky viewing) observed from morning to noon are associated to the fact that the Ci clouds responsible of sun occultation (strong solar attenuation) can be out from the field of view of the lidar system at that given moment.

\section{CONCLUSIONS}

A case study of $\mathrm{Ci}$ clouds observed over the Atmospheric Observatory 'El Arenosillo' (ARN) along the day (unfortunately, only lidar data until noon) has been examined. In general, LR and CCOD present an apparent opposite relationship: higher CCOD values correspond to lower LRs. The direct solar component is the most affected radiation by $\mathrm{Ci}$ presence. A good agreement is achieved between lidar and radiation data: dense opCi occurrence with a CCOD $\sim 2$ is observed in the interval 10:30-11:30 UTC when radiation components are strongly affected. Differences found between measurements and simulations of the surface solar radiation can be related to the particular sun occultation situation and the cloud cover portion as seen by the solar instrumentation and the field of view of the lidar system. Further $\mathrm{Ci}$ cases are to be examined in a future work.

\section{ACKNOWLEDGEMENTS}

This work was supported by the Spanish Ministerio de Economía y Competitividad (MINECO) under grants CGL2014-55230-R (AVATAR) and CGL2014-56255-C2-2-R (ICARO).

\section{References}

[1] Myhre et al., 2013: Anthropogenic and natural radiative forcing. In: Stocker, T.F., Qin, D., Plattner, G.-K., Tignor, M., Allen, S.K., Boschung, J., Nauels, A., Xia, Y., Bex, V., Midgley, P.M. (Eds.), Climate Change 2013: The Physical Science Basis. Contribution of Working Group I to the Fifth Assessment Report of the Intergovernmental Panel on Climate Change. Cambridge University Press, Cambridge, NY, USA.

[2] Flynn et al., 2007: Novel polarization-sensitive micropulse lidar measurement technique, Optics Express, 15 (6), 2785-2790.

[3] Córdoba-Jabonero et al., 2017: Diversity on subtropical and polar cirrus clouds properties as derived from both ground-based lidars and CALIPSO/CALIOP measurements, Atmos. Res., 183, 151-165.

[4] Dahlback and Stammes, 1991: A new spherical model for computing the radiation field available for photolysis and heating at twilight, Planet. Space Sci., 39, 671-683.

[5] Costa et al., 2014: Surface UV Radiation in the South of Portugal: Monitoring and Assessment of Cloud Effects, AIP Conference Proceedings, 04, 1531, 852 . 